\title{
A Low Emittance Lattice for the CLIC Damping Ring
}

\author{
J.P.Potier, CERN, CH-1211 Geneva 23, L.Rivkin, PSI, CH-5232 Villigen
}

\begin{abstract}
Damping rings with very small normalized equilibrium emittance and short damping times are required to deliver trains of bunches with a high repetition rate for the high-luminosity linear colliders. Using analytic expressions for the equilibrium emittance as a function of deflection angle per dipole, as well as the expression for the Twiss parameters providing the minimum emittance, a strategy to stay close to this minimum is described. In order to get as close as possible to the optimum Twiss parameters values, a quadruplet lattice with high horizontal phase advance is introduced. Finally, this approach is illustrated for the particular case of the CLIC damping ring and the resulting performance is described.
\end{abstract}

\section{INTRODUCTION}

The electron-positron linear colliders at high energy, requiring high luminosity, are all based on a high collision rate using beams of very small dimensions at the interaction point. These small beam dimensions require strong focusing in the final focus, as well as very small transverse emittance beams. Such small emittance will possibly be reached with high brightness electron guns presently under development, but is impossible to reach directly with the positron sources that have emittance three or more orders of magnitude higher. Therefore, for the positron beam, a damping ring facility will be necessary.

Most of the damping rings proposed nowadays are based on the Theoretical Minimum Emittance lattice (TME) in which the Twiss functions $\beta_{\mathrm{x}}$ and $D_{\mathrm{x}}$ are minimum in the middle of the bending magnet. The simplest example is a combined function bending magnet with a defocusing gradient and surrounding focusing quadrupoles to produce the appropriate Twiss functions. Such a lattice was proposed [1] for the CLIC damping ring. This ring was designed for the working energy of 3 $\mathrm{GeV}$ in order to get enough damping, but due to the strong chromaticity correction sextupoles, it suffered from a severe detuning versus emittance and from a great sensivity of the transverse dynamical acceptance to misalignment errors.

In this paper, using analytic expressions of the equilibrium emittance as a function of the deflecting angle per dipole, as well as the expression for the Twiss parameters corresponding to the minimum emittance, an expression for the emittance of the de-tuned lattice is proposed and a strategy to stay as close as possible to the minimum is developed, keeping the dispersion as high as possible, in order to limit the strength of the chromaticity correction sextupoles. A main damping ring based on this approach is then derived providing beam characteristics fulfilling, without any wigglers, the 500 GeV/c CLIC injector complex requirements [2]. The corresponding parameter list for this damping ring is summarised in Table 1.

\section{MINIMUM EMITTANCE LATTICE}

The transverse equilibrium beam emittance of a ring working at an energy $E$, composed of dipole magnets of length $L$ and deflection angle $\theta$, can be expressed as a function of the optics parameters in the middle of the bending magnet $\alpha, \beta, D, D$ '. After integration of the synchrotron integrals, using the approximation of small bending angle, the emittance is [3].

$$
\varepsilon_{x 0}=\frac{C_{q} E^{2}}{J_{x}} \frac{I_{5}}{I_{2}}=\frac{C_{q} E^{2}}{J_{x}}\left[\begin{array}{l}
\frac{\gamma D^{2}+2 \alpha \cdot D \cdot D^{\prime}+\beta D^{\prime 2}}{L} \cdot \theta \\
-\left(\frac{\alpha \cdot D^{\prime}+\gamma \cdot D}{12}\right) \cdot \theta^{2} \\
+\left(\frac{\beta}{12 \cdot L}+\frac{\gamma \cdot L}{320}\right) \cdot \theta^{3}
\end{array}\right]
$$

where $C_{q}=1.468 \cdot 10^{-6}\left[\frac{\mathrm{m}}{\mathrm{GeV}^{2}}\right]$. As is well known, this expression is minimum for $\alpha_{\mathrm{opt}}=D_{o p t}^{\prime}=0$ i.e. the optical and dispersion functions are symmetric with respect to the middle of the bending magnet and

$\beta_{o p t}=\frac{L}{2 \cdot \sqrt{15}}, D_{o p t}=\frac{L \cdot \theta}{24}, \varepsilon_{x 0}=\frac{C_{q} \cdot E^{2}}{J_{x}} \cdot \frac{\theta^{3}}{12 \sqrt{15}}$

For the detuned lattice, still preserving the symmetry, the emittance blow-up $\varepsilon / \varepsilon_{\text {opt }}$ can be expressed using the normalised values $\beta_{\mathrm{r}}=\beta / \beta_{\text {opt }}$ and $D_{\mathrm{r}}=\mathrm{D} / \mathrm{D}_{\mathrm{opt}}$

$$
\varepsilon_{r}=\frac{5}{8} \cdot\left[\frac{D_{r}^{2}}{\beta_{r}}-\frac{2 \cdot D_{r}}{\beta_{r}}\right]+\frac{9}{2} \cdot\left[\frac{1}{4 \cdot \beta_{r}}+\frac{\beta_{r}}{9}\right] .
$$

The family of curves for different values of constant blow-up ranging from 2 to 128 in the $\beta_{\mathrm{r}}, \mathrm{D}_{\mathrm{r}}$ diagram is shown using logarithmic scales in Figure 1.

For a given emittance blow-up, the maximum of $D_{r}=f\left(\beta_{\mathrm{r}}\right)$ curve is chosen, where

$$
\beta_{r m x}=\varepsilon_{r} \text { and } D_{r m x}=1+\frac{2}{\sqrt{5}} \cdot \sqrt{\varepsilon_{r}^{2}-1} \text {. }
$$

The curve representing the position of these maxima is also shown in Figure 1. 


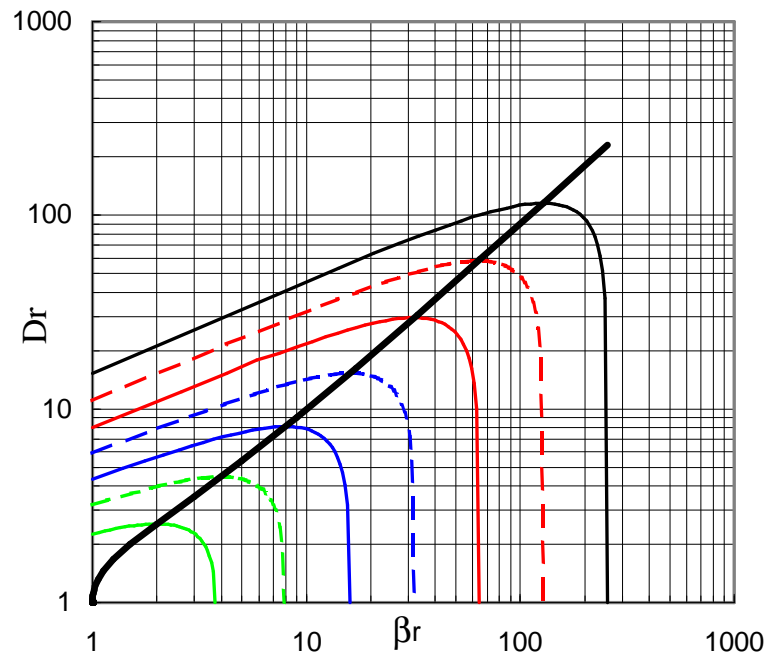

Figure 1 Dispersion versus beta function for a given emittance detuning and dispersion versus emittance for a maximum dispersion and a given emittance detuning

\section{CHOICE OF THE LATTICE PARAMETERS}

\subsection{Bending field and design energy}

In the CLIC injector complex [2], one train of 1 to 10 bunches is injected and extracted at a repetition rate, $f_{\mathrm{r}}$ from 1210 to 2400 , but a number of trains $k$, are concurrently circulating in the damping ring in order to leave a larger time for damping $T=k / f_{r}$. The present design is based on trains of 10 bunches, spaced by $20 \mathrm{~cm}$, at a repetition frequency $f_{r}$ of $1800 \mathrm{~Hz}$.

The damping ring circumference $C$ is chosen to accommodate the $k$ trains, with enough separation between them to allow for the rise and fall of the injection and extraction system $l_{\mathrm{k}}$. In the case of a fast kicker, $l_{k}$ is $7.5 \mathrm{~m}$ (25 ns). Using a particular combination of fast kickers and RF kickers, the time separation between the bunch trains, $l_{s}$ can be reduced down to $l_{k} / 2(12.5 \mathrm{~ns})$ [4]. If $n$ is the number of damping time necessary to damp the incoming emittance the reduced damping time

$\tau_{r}=\frac{\tau_{x, y}}{C} \quad$ has to satisfy the condition $\tau_{r} \leq \frac{1}{n \cdot l_{s} \cdot f_{r}}$

As shown in [4], the round beam produced by the positron injector is first pre-damped in a collector ring with large transverse and longitudinal acceptances during $5 \tau_{\mathrm{x}, \mathrm{y}}$ and then damped in the main damping ring (MDR) during $4 \tau_{\mathrm{x}, \mathrm{y}}$. Under these conditions, with $\tau_{\mathrm{r}} \leq 37 \mu \mathrm{s} / \mathrm{m}$, the beam emittance at extraction will not be larger than the equilibrium emittance by more than $10 \%$. This value, which depends on the bending field and on the design energy, can be written as

$$
\tau_{r}=\frac{2.54-x .10^{14}}{J_{x} \cdot B \cdot E^{2}}
$$

with $J_{\mathrm{x}}=1$ as the bending magnet will have no gradient. In order to maximise the damping, keeping the normalised emittance low, the highest possible field in the bending magnet is chosen while staying far from saturation, $B$ around $1.5 \mathrm{~T}$, and then $E$ from the above equations is $E=2.15 \mathrm{GeV} / \mathrm{c}$.

\subsection{Twiss functions in the middle of the bending magnets needed to produce the design emittances}

Taking into account the emittance blow-up between the ring exit and the interaction point, the normalised transverse emittances at the output of the main damping ring have to be $\gamma \varepsilon_{\mathrm{x}}=2.5 \cdot 10^{-6} \mathrm{radm}, \gamma \varepsilon_{\mathrm{y}}=0.04 \cdot 10^{-6} \mathrm{radm}$ with a bunch length $\sigma_{\mathrm{z}}=18 \mathrm{~mm}$. We have chosen to use a low emittance detuning value $\varepsilon_{\mathrm{r}}=3.7$ to use the TME lattice efficiently with $D_{r}=4.2$ close to the maximum on the detuning curve and $\beta_{\mathrm{r}}=4.4$ (see figure 1). The number $n_{\mathrm{B}}$ of cells can be worked out from the optimum equilibrium emittance and the emittance detuning, resulting in $n_{\mathrm{B}}=60$ and a dipole magnet length $L=.5 \mathrm{~m}$.

\section{QUADRUPLET TME LATTICE}

In order to provide the low dispersion and beta values in the middle of the dipole magnet, it will be flanked on each side by a defocusing quadrupole followed by a focusing quadrupole. The corresponding lattice is shown in Figure 2.

\begin{tabular}{|l|l|l|l|}
\hline Parameter & $\begin{array}{l}\text { Sym- } \\
\text { bol }\end{array}$ & Units & $\begin{array}{l}\text { TME } \\
\text { lattice }\end{array}$ \\
\hline Momentum & $E$ & $\begin{array}{l}\mathrm{GeV} / \\
\mathrm{c}\end{array}$ & 2.15 \\
\hline Number of cells & $\theta$ & $\mathrm{dg}$ & 60 \\
\hline Momentum compaction & $\alpha_{\mathrm{p}}$ & $10^{-3}$ & 0.29 \\
\hline $\begin{array}{l}\text { Normalised equilibrium } \\
\text { emittance }\end{array}$ & $\begin{array}{l}\gamma \varepsilon_{\mathrm{n}} \\
\mathrm{radm}^{-6}\end{array}$ & 2.56 \\
\hline Normalised damping time & $\tau_{\mathrm{r}}$ & $10^{-6} \mathrm{~s}$ & 37 \\
\hline $\begin{array}{l}\text { Horizontal phase advance per } \\
\text { cell }\end{array}$ & $\mu_{\mathrm{x}}$ & $2 \pi$ & 0.445 \\
\hline Vertical phase advance per cell & $\mu_{\mathrm{v}}$ & $2 \pi$ & 0.217 \\
\hline Emittance detuning $\varepsilon / \varepsilon_{\text {opt }}$ & $\varepsilon_{\mathrm{r}}$ & - & 3.7 \\
\hline $\begin{array}{l}\text { Dispersion in the middle of the } \\
\text { bending }\end{array}$ & $D$ & $\mathrm{~mm}$ & 9.4 \\
\hline$\beta$ in the middle of the bending & $\beta$ & $\mathrm{m}$ & 0.292 \\
\hline$\beta$ detuning & $\beta \mathrm{r}$ & - & 4.42 \\
\hline Dispersion detuning & $D_{\mathrm{r}}$ & - & 4.19 \\
\hline
\end{tabular}

Table 1: Main characteristics of the CLIC TME lattice

The main characteristics of a ring built of 60 cells are shown in Table 1. The values found are in good agreement with the analytical calculation made above. 


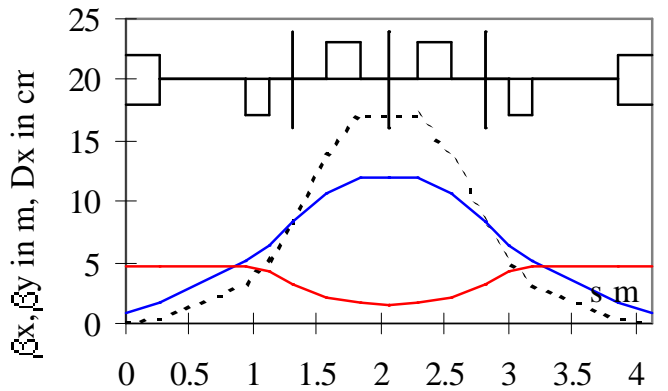

Figure 2: Twiss functions for the TME lattice providing an emittance detuning of 3.7

\section{MAIN DAMPING RING DESIGN}

Each damping ring arc consists of five TME quadruplet cells and two dispersion suppressers, that utilise half of a regular bending magnet. The arcs are separated by short straight sections equipped with quadrupole triplets to get $\alpha_{x, y}=0$ and a small $\beta_{x}$ in the middle. These short straight sections can house a damping wiggler. An arc is represented in Figure 3.

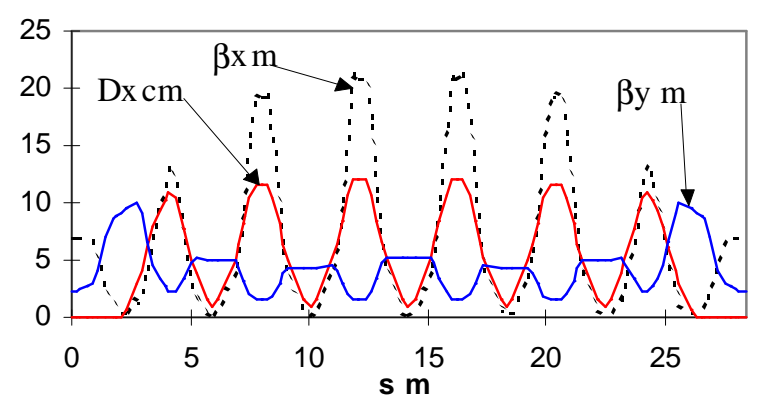

Figure 2: Arc of the CLIC main damping ring including short straight sections

The ring is made up of two halves, 5 arcs each, and two long straight sections with $\pi$ phase advance in each plane. These straight sections are inserted symmetrically, one for the RF cavities the other for the injection and extraction systems. The chromaticity is corrected by two families of sextupoles in the regular cells, and the 4 other families in the dispersion suppresser and in the straight sections.

The dynamic acceptance has been studied in the ring without the long straight sections. The closed orbit is corrected to $50 \mu \mathrm{m}$, using position monitors (assumed resolution $25 \mu \mathrm{m}$ ) located at each sextupole, with the correcting dipoles located at the same positions. The dynamic acceptance is more than $6.5^{*} 10^{-6}$ radm without errors. In this case, detuning versus the emittance in the aperture is less than 0.005 in the horizontal plane and 0.003 in the vertical plane at $10 \%$ coupling. In presence of errors, at full coupling, with $\mathrm{dP} / \mathrm{P}$ within $\pm 0.5 \%$ and position errors up to $50 \mu \mathrm{m}$, the dynamic acceptance is $4.0 * 10^{-6} \mathrm{radm}$.

As the bunch distance is a multiple of $0.2 \mathrm{~m}$ the RF frequency of the accelerating cavity will be $1.5 \mathrm{GHz}$ with a voltage of $1 \mathrm{MV}$ providing enough Touschek life-time. The parameters of the main damping ring are in Table 2.

\begin{tabular}{|l|l|l|l|}
\hline Parameter & $\begin{array}{l}\text { Sym- } \\
\text { bol }\end{array}$ & Units & $\begin{array}{l}\text { MDR } \\
\text { ring }\end{array}$ \\
\hline Momentum & $E$ & $\mathrm{GeV} / \mathrm{c}$ & 2.15 \\
\hline Number of cells & $\theta$ & $\mathrm{dg}$ & 60 \\
\hline $\begin{array}{l}\text { Ring length (without straight } \\
\text { sections) }\end{array}$ & $L$ & $\mathrm{~m}$ & 283 \\
\hline $\begin{array}{l}\text { Normalised equilibrium } \\
\text { emittance }\end{array}$ & $\gamma_{\mathrm{n}}$ & $\begin{array}{l}10^{-6} \\
\mathrm{radm}\end{array}$ & 2.5 \\
\hline Emittance detuning $\varepsilon / \varepsilon_{\mathrm{opt}}$ & $\varepsilon_{\mathrm{r}}$ & - & 3.8 \\
\hline Damping partition numbers & $J_{\mathrm{x}, \mathrm{y}}$ & & $1,1,2$ \\
\hline$\beta$ detuning & $\beta \mathrm{r}$ & & 4.4 \\
\hline Dispersion detuning & $\mathrm{Dr}$ & & 4.2 \\
\hline Normalised damping time & $\tau_{\mathrm{r}}$ & $10^{-6} \mathrm{~s}$ & 37 \\
\hline Bending magnet field & $B$ & $\mathrm{~T}$ & 1.47 \\
\hline Horizontal / vertical tunes & $\mu_{\mathrm{x}, \mathrm{y}}$ & $2 \pi$ & $31.2 / 1$ \\
\hline $\begin{array}{l}\text { Horizontal/vertical } \\
\text { chromaticities }\end{array}$ & $\mu_{\mathrm{y}}$ & & $-107 /$ \\
\hline Momentum compaction & $\alpha \mathrm{p}$ & $10^{-3}$ & 0.24 \\
\hline $\begin{array}{l}\text { Dynamical acceptance in }+/- \\
1 \%\end{array}$ & $A_{\mathrm{dyn}}$ & $\begin{array}{l}10^{-6} \\
\mathrm{radm}\end{array}$ & 4.0 \\
\hline Rms displacement errors & $\sigma_{\mathrm{misal}}$ & $\mu \mathrm{m}$ & 50 \\
\hline RF frequency & $\mathrm{f}_{\mathrm{r}}$ & $\mathrm{GHz}$ & 1.5 \\
\hline RF voltage & $V_{\mathrm{RF}}$ & $\mathrm{MV}$ & 1.0 \\
\hline Energy loss per turn & $U_{0}$ & $\mathrm{MV}$ & 0.39 \\
\hline Bunch length & $\sigma_{\mathrm{e}}$ & $\mathrm{mm}$ & 1.8 \\
\hline
\end{tabular}

Table 2: Main characteristics, of the main damping ring adapted to the CLIC requests using the TME lattice

\section{Conclusions}

Using an analytic approach, a damping ring working close to the theoretical minimum emittance has been designed fulfilling CLIC's requirements. As the CLIC bunch train length and repetition rate are evolving, this approach will be used to adapt the parameters and possibly introduce wigglers, to adapt to the new requests.

\section{References}

[1] J.P. Delahaye, J.P. Potier, Reverse Bending Magnets in a Combined-Function Lattice for the CLIC Damping Ring, CERN/PS 89-21 (LP), PAC, Chicago 1989.

[2] R. Bossart, H. Braun, F. Chautard, M. Comunian, J.P. Delahaye, J.C. Godot, I. Kamber, J.H.B. Madsen, L. Rinolfi, S. Schreiber, G. Suberlucq, I. Wilson, W. Wuensch, CLIC - Test Facility Developments and Results, CERN/PS 95-11 (LP), PAC, Dallas, 1995

[4] L.C. Teng , LS-17 1985, ANL-FNAL

[5] J.P. Delahaye and J.P. Potier, A Novel Injection and Extraction Scheme for Short Bunch Separation in Accelerator Rings, CERN/PS 94-15 (LP), EPAC, London, 1994

[6] J.P.Delahaye, An Injector Complex for the Main Beams of the CERN Linear Collider, CERN/PS 94-14 (LP), EPAC, London, 1994 